\title{
A comparison of lightning data provided by ZEUS and LINET networks over Western Europe
}

\author{
K. Lagouvardos ${ }^{1}$, V. Kotroni ${ }^{1}$, H.-D. Betz ${ }^{2}$, and K. Schmidt ${ }^{2}$ \\ ${ }^{1}$ National Observatory of Athens, Institute for Environmental Research, Athens, Greece \\ ${ }^{2}$ Nowcast GmbH, Munich, Germany
}

Received: 12 April 2009 - Revised: 20 August 2009 - Accepted: 30 August 2009 - Published: 19 October 2009

\begin{abstract}
In the framework of this paper, one-year of lightning data from the experimental network ZEUS operated by the National Observatory of Athens is compared to collocated data provided by the LINET detection network. The area of comparison is limited to a part of Central-Western Europe, where LINET data exhibits the highest data quality, permitting thus to be used as the validation dataset. The location error of ZEUS was calculated to be $\sim 6.8 \mathrm{~km}$, while the detection efficiency was $\sim 25 \%$, with a characteristic underdetection during nighttime. Moreover, the analysis revealed that ZEUS is also capable to detect not only cloud-to-ground but also intra-cloud strokes. Analysis of a specific case study revealed that the spatial distribution of ZEUS was very close to that of LINET, although the total number of strokes as seen by ZEUS is much lower than the one from LINET. The overall analysis permitted to assess the main characteristics of ZEUS network, information considered of paramount importance before the use of ZEUS data for a variety of observational and modeling work.
\end{abstract}

\section{Introduction}

Lightning has received a lot of attention in the scientific literature, since it is associated with severe storms that may cause considerable damages to agriculture, electric power networks, property and life. The Mediterranean Sea produces one of the major centers of electrical activity during the Northern Hemisphere winter (Christian et al., 1999). A number of recent studies on lightning in the Mediterranean are focusing on their spatiotemporal distribution (e.g. Altaratz et al., 2003; Katsanos et al., 2006), showing the preponderance of lightning activity over the Mediterranean waters

Correspondence to: K. Lagouvardos (lagouvar@meteo.noa.gr) during winter and over the continental Europe over spring and summer.

The Mediterranean area is covered by many national lightning detection networks that present good location accuracy but limited geographical coverage. Today, lightning data over broader areas is obtained by long-range detection systems such as the UK Meteorological Office Arrival Time Difference (ATD) system (Keogh et al., 2006), the World Wide Lightning Location Network (WWLLN, Rodger et al., 2005) as well as the National Observatory of Athens ZEUS detection system (Kotroni and Lagouvardos, 2008). Since high quality data provided by such systems are a prerequisite for the realization of a multitude of meteorological studies related to lightning, the performance assessment of these systems is an important task.

The aim of this paper is to exploit the capabilities of ZEUS lightning detection system to accurately detect lightning over Europe and the Mediterranean. A first inter-comparison of ZEUS and UK ATD system (Kotroni and Lagouvardos, 2008) during the summer period of year 2006 showed that ZEUS underestimates the nighttime and early morning activity both over land and sea. During the rest of the day ZEUS detects a larger number of lightning compared to ATD, attributable to the increased detection efficiency of ZEUS compared to ATD. Since in this study ZEUS was compared to another long-range detection network with known limitations as it concerns the detection efficiency and the location accuracy, a further comparison was necessary, this time with LINET, which is able to detect cloud-to-cloud and intracloud discharges (IC, hereafter), as well as cloud-to-ground (CG, hereafter) with a location accuracy of a few hundreds of meters. The period of comparison spans over one year (November 2007-October 2008) and is limited to central and southern Germany as well as parts of Austria and Switzerland, where the large number of deployed LINET sensors guarantees particularly high efficiency and accuracy (Betz et al., 2009).

Published by Copernicus Publications on behalf of the European Geosciences Union. 
The verification of ZEUS lightning detection system is considered as an important task, since lightning data are used for a multitude of applications, such as continuous monitoring of thunderstorm activity for the prompt protection of life and property. Moreover, lightning data are recently used as an additional tool for other meteorological applications, such as the improvement of satellite precipitation estimates, assimilation of lightning data to mesoscale modeling, nowcasting of severe weather, etc. (Mansell et al., 2007; Pessi and Businger, 2009). For that reason, the knowledge of the capabilities and the measurement limitations of a lightning detection system are considered particularly important.

The subsequent part of the paper is organized as follows: Sect. 2 presents the main characteristics of ZEUS and LINET, while results of the comparison are discussed in Sect. 3. Section 4 presents briefly a case study with significant lightning activity over the domain of comparison, while the last section draws the concluding remarks of this work.

\section{Data}

\subsection{ZEUS lightning detection network}

ZEUS is a long-range lightning detection network with receivers located at six sites over Europe (Birmingham in UK, Roskilde in Denmark, Iasi in Romania, Athens in Greece, Larnaka in Cyprus and Lisbon in Portugal). The system was manufactured by Resolution Displays Inc. ZEUS receivers record the radio noise (sferics) emitted by lightning strokes in the very-low-frequency range (between $7-15 \mathrm{kHz}$ ). The VLF signal is preamplified at each receiver site and the signal is synchronized to GPS time. At each receiver site an identification algorithm is executed that detects a probable sferics candidate, excluding weak signals and noise, and is capable of capturing up to 70 sferics per second. Then, the lightning location is retrieved at the central station of the network using the arrival time difference triangulation technique. The arrival time difference values represent positions between two stations with the same time difference, and their intersection defines a sferic fix. ZEUS locating algorithm requires a minimum of four receivers to record the same event. Further details on ZEUS locating algorithm can be found in Chronis and Anagnostou (2006). Real time ZEUS data over Europe and the Mediterranean Sea can be found at http://www.www.noa.gr/forecast/lightning.gif (maps are updated every $15 \mathrm{~min}$ ).

In the frame of this study ZEUS data from November 2007 up to October 2008 are analyzed. Although ZEUS is an experimental network, the data availability during this period was quite high (97\%), therefore, the $3 \%$ gaps within the dataset are not expected to have significant impact on the results presented in the following.

\subsection{LINET lightning detection network}

LINET network consists of $\sim 100$ sensors across 20 European countries, with some 30 sensors within Germany. LINET station locations can be found at https://www. nowcast.de. LINET exploits the VLF/LF regime and combines the measurement of CG and IC discharges. IC strokes are discriminated against CG strokes as long as the lightning occurs within $\sim 120 \mathrm{~km}$ from the nearest sensor. The domain chosen for the comparison against ZEUS data guarantees a network geometry that allows good IC identification. According to Betz et al. (2009), LINET offers an optimized location accuracy reaching an average value as small as $\sim 150 \mathrm{~m}$, verified by strikes into towers of known position, so that LINET data can be considered as a valuable "ground truth" dataset for the evaluation of ZEUS data.

\section{ZEUS and LINET data comparison}

The comparison between ZEUS and LINET networks deals exclusively with strokes, since the aim is to compare the very basic detection capabilities of two different systems. Table 1 gives an overview of the two analyzed dataset from both ZEUS and LINET networks. During the one-year verification period and over the study area, LINET and ZEUS sensed 4792104 and 1099546 strokes, respectively. The time coincident events within $\pm 1 \mathrm{~ms}$ are 770218 . From the analysis of the time coincident events it was evident that ZEUS can also detect IC strokes, a fact considered very important for the detection of thunderstorm activity that is predominantly characterized by IC activity. A similar result was also found on the analysis of WWLLN data over Florida (Jacobson et al., 2006). From Table 1 it is evident that the percentage of IC strokes relative to the total number of strokes sensed by LINET is $\sim 57 \%$, while for the coincident strokes it is only $\sim 35 \%$, indicating thus that ZEUS detects IC with a reduced efficiency compared to CG strokes.

\subsection{Location error}

Figure 1 presents the distribution of the location difference of time-coincident strokes. Inspection of Fig. 1 as well as analysis of the corresponding data reveals the following:

- The distribution peaks at a location difference of $5 \mathrm{~km}$.

- 75\% of the collocated strokes have an error less than $12 \mathrm{~km}$, while the $90 \%$ of the strokes have an error less than $18 \mathrm{~km}$. Above this threshold, the number of strokes diminishes very fast.

- The mean error of the stroke locations amounts to $\sim 6.8 \mathrm{~km}$.

Therefore, ZEUS provides measurements that present a small location error, taking into account that it is a long-range 


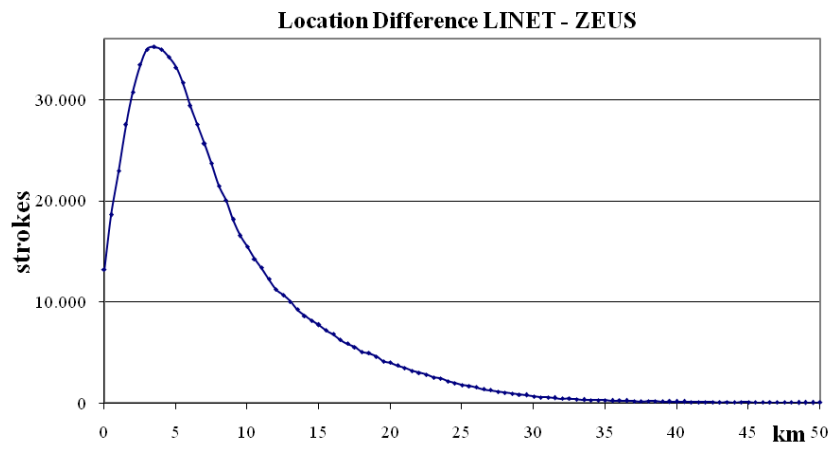

Fig. 1. Location difference between LINET and ZEUS strokes.

detection system. For comparison, WWLLN system (at its configuration in 2004) presented a location error of $\sim 15 \mathrm{~km}$ over Florida (Jacobson et al., 2006), while ATD presents a location error over Europe of $\sim 20 \mathrm{~km}$ (Keogh et al., 2006).

\subsection{Detection efficiency}

The efficiency of a lighting detection network to record the maximum possible number of strokes over an area is also of great importance. This efficiency can be measured not only as the fraction of strokes seen by both systems but also as the ability of one long range detection system as ZEUS to locate thunderstorm activity even with reduced number of strokes. This twofold approach of the problem is treated in the following sections.

Figure 2 presents the detection efficiency of ZEUS (expressed in percent with respect to LINET strokes) for the 40 days of the most intense lightning activity over the area (black bars). Since the study area is over a continental area, the majority of these 40 days occurred during summer (all cases, except one, occurred from May to September 2008). As can be seen, the detection efficiency varies from day-today, with a mean efficiency of $\sim 25 \%$ for these forty days. Extending the estimation of the mean location efficiency throughout the year, this number remains the same.

As stated in the introduction, Kotroni and Lagouvardos (2008) have performed a comparison of ZEUS and ATD lightning detection system for a 3-month summer period and concluded that during the night-time (especially between 21:00 and 03:00 UTC) ZEUS presents a tendency to underdetect lightning. For that purpose, the calculation of the detection efficiency was repeated for the ZEUS data, but now only for the period of the day between 21:00 and 03:00 UTC (grey bars in Fig. 2). It is clear that ZEUS efficiency drops for all days during nighttime, with a mean detection efficiency of $\sim 9 \%$. However, since during these nighttime hours, the number of strokes (as sensed by LINET) is limited (only 11\% of the total daily number of strokes), this under-detection of strokes by ZEUS, although a deficiency of the system, is

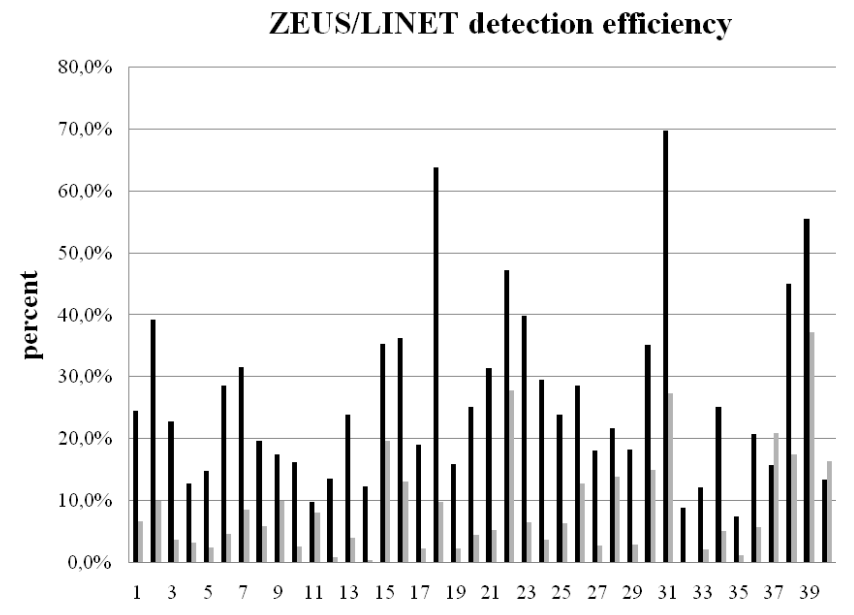

Fig. 2. ZEUS/LINET detection efficiency for the 40 days with the most significant activity over the study area. Solid black bars denote daily data, while grey bars data for the period between 21:00-03:00 UTC.

Table 1. Events during the one-year period.

\begin{tabular}{ll}
\hline LINET events & 4792104 \\
ZEUS events & 1099546 \\
LINET events per type (CG/IC) & $2089892 / 2702212$ \\
Time-coincident events & 770218 \\
Time-coincident events per type (CG/IC) & $504616 / 265609$ \\
\hline
\end{tabular}

not considered as very important for lightning occurring over land. It can be however a shortcoming for the study of lightning over the sea, where lighting activity does not show any clear day/night preference. The reason for this behavior of ZEUS system is not very clear, a possible explanation could be the fact that thunderstorm activity over remote areas as South America during their local day hours (night hours in Europe) may produce stronger signal than the strokes over Europe leading thus to underestimation of the number of strokes detected over Europe during the night hours.

\section{The 30 May 2008 case-study}

It should be interesting at this stage to investigate more closely one case study and for that purpose the day with the largest number of strokes, as detected by LINET, namely on 30 May 2008, was selected. During this day, a lowpressure system moving over the southern part of Germany created the necessary instability for the onset of severe thunderstorms in the area. LINET detected during the day more than 390000 strokes, with $\sim 75000$ strokes within one hour (17:00-18:00 UTC). During the same day, ZEUS detected 96000 strokes, with $\sim 15000$ strokes during the same hour. 


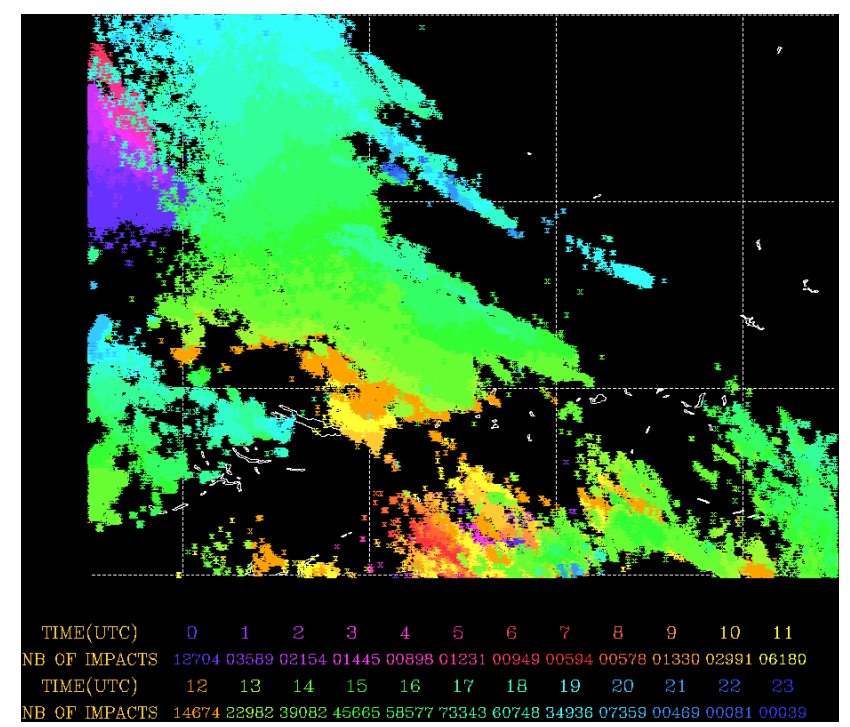

Fig. 3a. LINET strokes, plotted every hour over the analyzed area.

Figure 3 shows the hourly distribution of lightning, as sensed by each network. Two important results are revealed by inspection of this figure: (a) ZEUS, as expected, clearly underestimates the number of strokes each hour, with about $24 \%$ of detection efficiency during the period of maximum lightning intensity (14:00-19:00 UTC), (b) the spatial distribution of lightning, as sensed by the two networks, reveals that the major centers of lightning activity appear similar, with great overlap in time and space.

As discussed earlier in the beginning of Sect. 3.2, the detection efficiency can be also seen not only as a simple fraction of strokes detected ( $24 \%$ for this case) but also as the ability of ZEUS to detect at least one lightning related to a thunderstorm. For that purpose, the study area (a rectangle box from $7^{\circ} \mathrm{E}$ to $15^{\circ} \mathrm{E}$ and from $46^{\circ} \mathrm{N}$ to $51.5^{\circ} \mathrm{N}$ ) has been divided in square boxes (sized $0.1^{\circ} \times 0.1^{\circ}$, each box approximately corresponds to an area of $\sim 75 \mathrm{~km}^{2}$ ). Therefore, a grid of $81 \times 56$ grid boxes was created and the total number of lightning, as sensed by each network, was counted within each one of the 4536 grid boxes. Further, a $2 \times 2$ contingency table was created, as shown in Table 2, where LINET strokes are considered as the ground truth and ZEUS data as the verifying dataset. In Table 2, A is the number of grid boxes for which LINET and ZEUS observed at least one stroke (hits), $\mathrm{B}$ is the number of grid boxes for which ZEUS detected at least one stroke but LINET did not (false alarm), $\mathrm{C}$ is the number of grid boxes with at least one stroke detected by LINET but not from ZEUS (misses) and D is the number of boxes where neither LINET nor ZEUS detected strokes (correct negatives).

From A, B, C and D given in Table 2, the following classical scores can be calculated, namely:

- Probability Of Detection, $\mathrm{POD}=\mathrm{A} /(\mathrm{A}+\mathrm{C})$

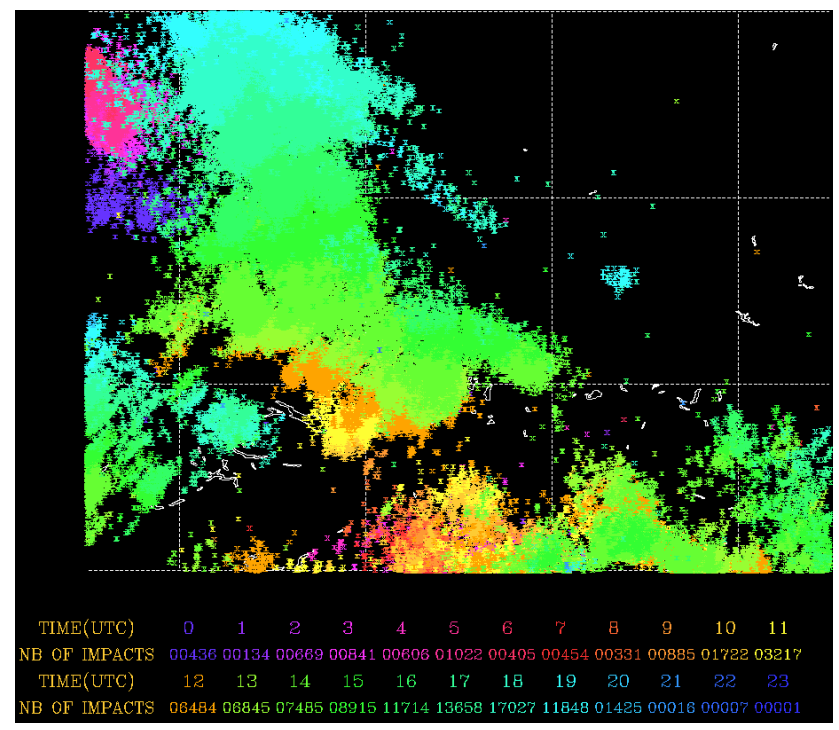

Fig. 3b. As in Fig. 3a but for ZEUS strokes.

Table 2. $2 \times 2$ contingency table.

\begin{tabular}{llll}
\hline & & Event observed by LINET \\
\hline & & Yes & No \\
Event observed by ZEUS & Yes & A & B \\
& No & C & D \\
\hline
\end{tabular}

- False Alarm Ratio, FAR=B/(A+B)

Application of the aforementioned calculations led to a POD of 0.90 and a FAR of 0.11 , results that can be considered as very satisfactory, showing the ability of ZEUS to detect the areas associated with lightning activity with a very high probability of detection. Therefore, although ZEUS detects in total $24 \%$ of LINET strokes, it can provide a very good spatial distribution of the areas affected by lightning, delineating thus efficiently the areas affected by thunderstorm activity. This finding is considered as an asset of a long-range detection system for many applications (thunderstorm monitoring, assimilation of lighting data in mesoscale models, etc.) for which the spatial distribution of lightning occurrence is required.

\section{Concluding remarks}

In the frame of this paper, the comparison of one-year lightning data provided by ZEUS lightning detection network against collocated LINET data was explored. The comparison was performed over an area comprising Central and 
Southern Germany as well as parts of Switzerland and Austria, where the dense LINET network can guarantee a high detectability of lightning strokes with location accuracy of the order of a few hundred meters.

The analysis of the location error revealed a mean error of $\sim 6.8 \mathrm{~km}$, a result that can be considered as very satisfactory for a long-range detection system, consisting from only 6 sensors across Europe. As it concerns the detection efficiency, the analysis of results showed that it is of $\sim 25 \%$, with a clear tendency from ZEUS to under-detect lightning during the night. ZEUS was also found able to detect IC strokes, although with a reduced detection efficiency compared to CG strokes. This characteristic is considered as an advantage since ZEUS is able to detect also those thunderstorms that are mainly accompanied by IC strokes and very few CG strokes.

Further, a detailed analysis of one day characterized by the largest number of lightning during the one-year verification period, revealed that although ZEUS detects about one stroke for every four detected by LINET, the spatial distribution of lightning within boxes of $0.1 \times 0.1$ deg revealed a very high POD and very low FAR. This finding means that ZEUS data can be used as a good indicator of lightning activity, presenting thus high storm-detection efficiency.

Since today ZEUS real-time data are used not only by the National Observatory of Athens but also by other European research centers for a variety of meteorological applications (including space-borne precipitation estimation, nowcasting of thunderstorm activity over the Mediterranean, etc.), the verification of ZEUS network will be a permanent task of the authors.

Acknowledgements. This work has been supported by the EU financed project FLASH (Contract No. 036852).

Edited by: S. Michaelides, K. Savvidou, and F. Tymvios Reviewed by: C. Price and another anonymous referee

\section{References}

Altaratz, O., Levin, Z., Yair, Y., and Ziv, B.: Lightning activity over land and sea on the eastern coast of the Mediterranean, Mon. Weather Rev., 131, 2060-2070, 2003.

Betz, H.-D., Schmidt, K., Laroche, P., Blanchet, P., Oettinger, P., Defer, E., Dziewit, Z., and Konarski, J.: LINET-an international lightning detection network in Europe, Atmos. Res., 91, 564573, 2009.

Christian, H. J., Blakeslee, R. J., Boccippio, D. J., Boeck, W. L., Buechler, D. E., Driscoll, K. T., Goodman, S. J., Hall, J. M., Koshak, W. J., Mach, D. M., and Stewart, M. F.: Global frequency and distribution of lightning as observed by the Optical Transient Detector (OTD), Proc. 11th Intl. Conf. on Atmospheric Electricity (ICAE), Guntersville, AL, 726-729, 7-11 June 1999.

Chronis, T. and Anagnostou, E.: Evaluation of a long-range lightning detection network with receivers in Europe and Africa, IEEE T. Geosci. Remote, 44, 1504-1510, 2006.

Jacobson, A. R., Holzworth, R., Harlin, J., Dowden, R., and Lay, E.: Performance assessment of the Worlds Wide Lightning Location Network (WWLLN), using the Los Alamos Sferic Array (LASA) as ground truth. J. Atmos. Ocean. Tech., 23, 1082-1091, 2006.

Katsanos, D., Lagouvardos, K., and Kotroni, V.: Combined analysis of rainfall and lightning data produced by mesoscale systems in the central and eastern Mediterranean, Atmos. Res., 83, 55-63, 2006.

Keogh, S. J., Hibbett, E., Nash, J., and Eyre, J.: The Met Office Arrival Time Difference (ATD) system for thunderstorms detection and lightning location, Forecasting Research, Techn. Rep. No. 488, 2006.

Kotroni, V. amd Lagouvardos, K.: Lightning occurrence in relation with elevation, terrain slope and vegetation cover in the Mediterranean, J. Geophys. Res., 113, D21118, doi:10.1029/2008JD010605, 2008.

Mansell, E., Ziegler, C., and MacGorman, D.: A lightning data assimilation technique for mesoscale forecast models, Mon. Weather Rev., 135, 1732-1748, 2007.

Pessi, A. T. and Businger, S.: The Impact of Lightning Data Assimilation on a Winter Storm Simulation over the North Pacific Ocean, Mon. Weather Rev., doi:10.1175/2009MWR2765.1, in press, 2009.

Rodger, C. J., Brundell, J. B., and Dowden, R. L.: Location accuracy of VLF World-Wide Lightning Location (WWLL) network: Post-algorithm upgrade, Ann. Geophys., 23, 277-290, 2005, http://www.ann-geophys.net/23/277/2005/. 